\title{
Flux canceling in three-dimensional radiative magnetohydrodynamic simulations
}

\author{
Irina Thaler ${ }^{1,2}$ and H. C. Spruit ${ }^{1}$ \\ 1 Max-Planck-Institut für Astrophysik, Karl-Schwarzschild-Str. 1, 85748 Garching, Germany \\ e-mail: thaler@leibniz-kis.de \\ 2 Kiepenheuer Institute for Solar Physics, Schöneckstraße 6, 79104 Freiburg im Breisgau, Germany \\ Received 28 February 2014 / Accepted 3 February 2017
}

\begin{abstract}
We aim to study the processes involved in the disappearance of magnetic flux between regions of opposite polarity on the solar surface using realistic three-dimensional (3D) magnetohydrodynamic (MHD) simulations. "Retraction" below the surface driven by magnetic forces is found to be a very effective mechanism of flux canceling of opposite polarities. The speed at which flux disappears increases strongly with initial mean flux density. In agreement with existing inferences from observations we suggest that this is a key process of flux disappearance within active complexes. Intrinsic $\mathrm{kG}$ strength concentrations connect the surface to deeper layers by magnetic forces, and therefore the influence of deeper layers on the flux canceling process is studied. We do this by comparing simulations extending to different depths. For average flux densities of $50 \mathrm{G}$, and on length scales on the order of $3 \mathrm{Mm}$ in the horizontal and $10 \mathrm{Mm}$ in depth, deeper layers appear to have only a mild influence on the effective rate of diffusion.
\end{abstract}

Key words. Sun: magnetic fields - Sun: photosphere - Sun: granulation

\section{Introduction}

The magnetic flux that emerges in new active regions throughout a sunspot cycle has largely disappeared from view again at the next minimum. The emergence of active regions at the surface is conspicuous even at moderate spatial resolution, but the process by which it disappears has proved to be much harder to observe. This is even more striking on shorter timescales. In active complexes, where new active regions continue to emerge within a limited region over periods from weeks to months, the total flux seen in synoptic magnetograms remains relatively stable. From observations of these kinds of regions Howard \& Labonte (1981) deduced that newly-erupted flux disappears from view in about ten days.

Direct observations of individual active complexes confirm this (Rabin et al. 1984; Kálmán 2001, are impressive examples). The implication is that magnetic flux elements of opposite polarities somehow meet each other within the confines of the complex and disappear together without noticeable signatures in the atmosphere. As stressed by Kubo et al. (2010a), this holds down to the smallest length scales accessible with Hinode/SOT (about $0.2 \operatorname{arcsec})$.

In descriptions of high-resolution observations this process is called "canceling" (Martin et al. 1985; Yang et al. 2012). The two-dimensional (2D; surface) description implied by this term does not do justice to the three-dimensional (3D) nature of magnetic fields. An interpretation in terms of "retraction" is physically more realistic here. (cf. Sect. 2.1).

Surface flux transport models (SFT) have been developed to account for the evolution of the surface magnetic field on larger scales (e.g., Jiang et al. 2014, and references therein). In these models the magnetic field is treated as a scalar field governed by a two-dimensional diffusion equation.
Flux emergence in newly-forming active regions looks almost like diffusion, but with the arrow of time reversed such that small scale mixtures of opposite polarity separate themselves out into large-scale bipolar active regions. In addition to the flux separation from a mix, the centers of polarity of the region also systematically seperate from each other. This continues after most of the flux has already emerged ${ }^{1}$. In a diffusion view, a negative value of the diffusion coefficient would have to be arbitrarily assumed in regions where flux is observed to be emerging. In an SFT model newly-emerging active regions are instead implemented by adding them as source terms in its diffusion equation. To prevent rapid canceling of emerging flux when an active region's polarities are still at a small distance, the sources are added at a more advanced stage, omitting their initial emergence and spreading phase.

Small-scale displacements of identifiable magnetic concentrations are used to derive observational values for diffusion coefficients. These displacements are dominated by granulation and supergranulation flows. The assumption is made that the evolution of average magnetic fields on larger spatial scales can be described by an effective diffusion based on these sorts of random displacements. On the scale of granular flows, the values obtained by "magnetic feature tracking" were $D=70-90 \mathrm{~km}^{2} \mathrm{~s}^{-1}$ from Solar and Heliospheric Observatory (SOHO)/Michelson Doppler Imager (MDI) magnetograms (Hagenaar et al. 1999). Somewhat lower values, $D$ $60 \mathrm{~km}^{2} \mathrm{~s}^{-1}$ were reported from higher resolution observations

\footnotetext{
1 This was noted early on as a characteristic property of active-region emergence and sunspot formation in general. Its demonstration requires time series such as those by Vrabec (1974, interpreted by Zwaan 1978). A well documented and more recent example is the "Hinode trilobite": http://science1.nasa.gov/media/medialibrary/2007/ 09/18/18sep_trilobite_resources/Hinode_lower.mov. Additional references are given further on in this section.
} 
using "bright point tracking" with the Swedish Vacuum Solar Telescope (Berger et al. 1998). Using the New Solar Telescope (NST) at the Big Bear Observatory, magnetic diffusivity values between around $20-300 \mathrm{~km}^{2} \mathrm{~s}^{-1}$ were obtained for a quiet sun region, depending on the spatial and temporal scale (Abramenko et al. 2011). For supergranular flows, the inferred diffusion coefficients range from $120-230 \mathrm{~km}^{2} \mathrm{~s}^{-1}$ using crosscorrelation techniques (Wang 1988; Komm et al. 1995) and 140-300 $\mathrm{km}^{2} \mathrm{~s}^{-1}$ using object tracking (Smithson 1973; Mosher 1977; Schrijver \& Martin 1990).

The evolution of large-scale magnetic patterns during the solar cycle is classically attributed to the effect of granulation and supergranulation (Leighton 1969). Diffusion coefficients required to match the dispersal of the large-scale field, of order $600 \mathrm{~km}^{2} \mathrm{~s}^{-1}$ (Sheeley 1992), differ from the small-scale measurements. Inclusion of the effect of a meridional circulation reduces the discrepancy (Wang et al. 1991). Even apart from the seemingly negative diffusion in emerging flux regions, the remaining variation in numbers suggests that other factors are at work besides the observed convective flows.

Because magnetic fields are divergence-free, field lines have no ends and are intrinsically 3D. In contrast with a scalar quantity sprinkled on a surface, field lines continue above and below it, and Lorentz forces are present along their entire length. It would be very surprising if the evolution of the surface magnetic field could be reduced to the effects of the flows observed at the surface. To the extent that field lines are anchored in deeper layers, their evolution is governed by conditions in those layers, rather than by surface observables. With the advent of realistic 3D magnetohydrodynamic (MHD) simulations of the solar photosphere, it has become possible to study the physics underlying the dispersal of magnetic fields in a much more comprehensive way than is possible from observations. As a step in this direction, in this work we study the dependence of "flux canceling" on conditions in the deeper layers of the simulation and on the mean strength of the field.

To quantify the canceling rates, we extract effective diffusion coefficients from the simulations, that is, numbers that in a simple 2D diffusion model would yield the same decay rates of unsigned flux. The actual 3D MHD evolution of the field configuration bears little relation to this sort of a simple view or to other turbulent diffusion models. This reflects itself in the measured values being far from constant and depending not only on time but also on all other factors such as horizontal length scale, depth of the simulation box, and the initial mean flux density. Our expectation is that the dependence on these factors will help, both in conceptualizing the processes at work, and in interpreting observed canceling rates. The need for length-scale-dependent effective diffusion coefficients is evident from the very large range of values measured, such as those in Berger et al. (1998), Chae et al. (2008) and Abramenko et al. (2011).

\section{Magnetic coupling between the solar surface and its interior}

If it were possible to model the displacements of field lines only by taking into account convective flows ("turbulent diffusion model"), then these models would need to accommodate negative as well as positive diffusion coefficients. However, this is in conflict with the MHD equations, which are invariant to a change of sign of the magnetic field vector. A consequence of this invariance is that there is no flow, however complex, that can separate magnetic polarities out of a random mixture.
The observations show flux fragments traveling systematically to the sunspot of their own polarity, ignoring all convective flows in the area (e.g., Vrabec 1974; Strous et al. 1996). The emergence process is driven by forces in the magnetic field itself. It is to be understood as the result of a bundle of magnetic field rising through the surface (as proposed first by Cowling 1953). Numerical simulations of a rising flux bundle (Isobe \& Shibata 2004; Cheung et al. 2008) reproduce the separation process convincingly. The separation process of the opposite polarities is a combination of the magnetic buoyancy bringing the field to the surface (the "rising tree" picture, Vrabec 1974; Zwaan 1978, 1985) and the unbalanced magnetic tension in the part of the horizontal field strand that remains below the surface (Moreno Insertis et al. 1994).

The difficulty of including the emergence process in models for surface field evolution, other than by ad hoc procedures, illustrates the importance of the connection of the surface field with its roots in deeper layers namely the 3D, solenoidal nature of the magnetic field. Given this connection, one should expect that it also plays a role in regions where no new flux emerges. It follows that the evolution of the large-scale field at the surface is not determined just by the observed displacements such as granulation, supergranulation, and meridional circulation, but also by barely observable processes deeper down.

The degree to which deeper layers contribute depends on the strength of their magnetic connection to the surface. This connection is determined by the intrinsic field strength of the magnetic concentrations, rather than an average (unsigned) flux density at the surface. At a strength of $50 \mathrm{G}$ the connection is weak, but at the intrinsic strength of magnetic concentrations, of the order 1-2 kG, the Alfvén speed is higher than convective flow speeds down to depths of several Mm. At these depths, the field strength in the magnetic elements may actually be even higher than at the surface, and the connection even stronger.

The size of the region over which the diffusion acts is also important. If the length scale on which the polarities are mixed is small, anchoring at a large depth is unlikely to affect the rate of canceling as seen at the surface. On the other hand, if the horizontal distance of interest is large, such as hundreds of Mm, anchoring effects as deep as the base of the convection can become relevant for the evolution of the surface field.

\section{1. "Flux canceling"}

This subsection contains some theoretical considerations relevant to the interpretation of the results reported below, in particular their dependence on the mean field strength, the so-called "average unsigned flux density" in Sect. 4.3.

\subsubsection{Mixing vs. canceling}

At low spatial resolution, a mixture of opposite polarities becomes undetectable long before the small-scale structures that make up the mix have physically disappeared. To detect physical disappearance from the surface, the spatial resolution has to be high enough to resolve the scale on which the process has an observational signature, beyond apparent in situ disappearance. Kubo et al. (2010b) do not detect horizontal fields between the polarities, and infer that the interaction leading to the actual disappearance takes place below the 0.2 -arcsec resolution of the Hinode/SOT instrument. The difference between mixing and canceling is academic in a turbulent diffusion view, in which the evolution of the field is seen as a cascading process of decreasing 
length scales. Guided by experience in ordinary hydrodynamics, the net rate of mixing and magnetic dissipation in this view is assumed to be independent of the length scale on which the actual reconnection takes place. This view would then apply when the field strength is low enough, such that Lorentz forces can be neglected compared with the characteristic hydrodynamic forces.

\subsubsection{The effect of field strength}

At the intrinsic $\mathrm{kG}$ strength of the small-scale magnetic field, magnetic forces cannot be ignored in the discussion of canceling. For example, high-resolution observations of small scale flux elements show that their displacements do not match the flows in the granulation around them (Kubo et al. 2010a).

The effects are different between the high- $\beta$ environment below the photosphere and the low- $\beta$ atmosphere. Below the surface, the magnetic elements are separated from each other by low-field-strength plasma; they do not exert forces on each other unless they are brought into direct contact.

This is different in the low- $\beta$ atmosphere. The Alfvén speed in the atmosphere of a typical active-region field strength of $50 \mathrm{G}$ is so high that reconnection is fast compared with the time scale on which the magnetic elements move around. As a result, the atmospheric field is in a quasi-static state close to the lowest energy configuration, meaning that it is a force-free field. Its field lines connect the two polarities through the atmosphere, as also evident from the morphology of structures seen in chromospheric lines.

\subsubsection{Retraction}

Because the patches about to cancel are already magnetically connected, the changes in magnetic energy associated with their approach do not turn up as dissipation in the atmosphere. Instead, they are accounted for by the work done against the forces acting at the foot points. This is similar to how the magnetic energy in the field around two neighboring bar magnets of opposite polarity ends up in the hands that keep them apart. This explains why only modest evidence of magnetic energy release in the atmosphere is found in canceling events ${ }^{2}$. Most likely, they are evidence of retraction. When the attraction has brought elements of opposite polarity together to within a distance of the order of their diameters, the force becomes strong enough for magnetic tension to pull the connecting field lines ("hairpins") below the surface. This process of "retraction" has been invoked intuitively as the main cause of the observed cancelation in mixed polarity regions (e.g. Rabin et al. 1984; Topka et al. 1986; Kálmán 2001). Because the attractive force increases as the square of the field strength, one should expect that effective diffusion coefficients used to model canceling will turn out to depend on the mean absolute field strength in the area (the "unsigned flux density") This is confirmed by the results reported in Sect. 4.3. The case of new flux erupting into an existing field differs from canceling in an area of mixed polarity. The field of a new bipole already reconnects to the pre-existing field of the atmosphere during

\footnotetext{
Poynting flux is sometimes discussed in the context of heating of the atmosphere. It is not a reliable measure of actual magnetic heating, however. The Poynting flux includes not only dissipation of energy, but also changes in magnetic energy content unrelated to dissipation. The magnetic energy content of the atmosphere above two neighboring flux tubes of opposite polarity decreases as the two are brought together. The associated Poynting flux is downward, the corresponding energy is delivered to the footpoints. See also Sect. 1.9 in Spruit (2013).
}

the emergence process itself. Where new flux erupts under the penumbral field overlying the photosphere it produces the socalled Ellerman bombs observed around sunspots and in emerging flux regions (EFR) between growing sunspots (Watanabe et al. 2011; Vissers et al. 2013; Peter et al. 2014). In the absence of a strong field to reconnect against, the magnetic energy of an erupting bipole ends up in the field configuration that it establishes in the atmosphere ${ }^{3}$.

\subsubsection{Canceling vs. reconnection}

Evidence of heating in the atmosphere in connection with canceling events, apart from the case of flux emergence into a preexisting field as discussed in the previous section, has been searched for extensively but with somewhat inconclusive results (Rabin 1984; Yang 2012). For example, Kubo et al. (2014) conclude that the canceling process does not leave detectable traces down to the Hinode resolution of 0 '!3. This is as expected from retraction (the text at Figs. 17-20 provides details).

This is not surprising, because the atmosphere does not react to canceling the same way it reacts to the emergence of new flux, such as the coronal bright points observed associated with emergence of small bipoles. The opposite polarities in a canceling event have already been around on the surface long before they meet. Their field lines are connected already. By this connection, they attract each other, most strongly on their final approach. The energy released in this way powers the downflow in retraction, rather than appearance in the atmosphere. The Poynting flux in a retraction event is downward. It is converted into the kinetic energy of the downflow, disappearing into the depths of the convection zone. Section 1.9 in Spruit (2013) contains more on this.

\subsection{Simulations}

In recent years there have been several attempts to measure effective turbulent diffusion coefficients in direct numerical simulations. For example, Hotta et al. (2012), determine turbulent diffusion coefficients following a passive density scalar in a 3D hydrodynamic simulation. They find a diffusion coefficient dependence on the vertical extent of the box. However, in the presence of magnetic fields the quantity of interest is a vector and not a scalar, which can undergo field amplification that will result in it backreacting on the flow due to the Lorentz force. Therefore one should be aware of the limits of the passive scalar approach compared to the evolution of a real MHD flow (further discussed in Sect. 5). The numerical, analytical and experimental difficulties of determining magnetic turbulent diffusion coefficients are discussed by Tobias \& Cattaneo (2013), who proposed a new measurement method for numerical simulations and laboratory experiments. Studying the decay of a sunspot, Rempel \& Cheung (2014) describe the process by using a constant turbulent magnetic diffusion coefficient of $350 \mathrm{~km}^{2} \mathrm{~s}^{-1}$ over a time span of two days.

Cameron et al. (2011) study the rate of canceling of opposite polarities at the surface using 3D radiative MHD simulations extending to $800 \mathrm{~km}$ below the surface. Their simulations start with a vertical field of uniform strength, in a two by two checkerboard pattern of positive and negative polarities. The simulations follow the decay of the (unsigned) flux density in an area

\footnotetext{
3 In the stronger fields erupting in emerging flux regions (EFR), kinetic energy associated with the unloading of mass from the rising field also plays some role. It manifests itself in downflows and brightenings at the magnetic footpoints (Shibata et al. 1989).
} 
of $6 \times 6 \mathrm{Mm}^{2}$. The diffusion coefficients obtained there depend critically on the boundary conditions used, and show a range of $D \sim 100-340 \mathrm{~km}^{2} \mathrm{~s}^{-1}$. For the most realistic boundary conditions, meaning a potential field boundary for the magnetic field and open boundaries for flows, similar to the boundary conditions used in this paper, they derive an effective diffusion coefficient of $D \sim 340 \mathrm{~km}^{2} \mathrm{~s}^{-1}$. This is then a diffusion rate that applies to field dispersal and cancelation, provided that only the flows down to a depth of the order of half a granule contribute, and that anchoring effects below this depth are absent.

With the calculations reported below, we measure effective diffusion rates in simulations extending to significantly greater depths below the surface. The purpose is to determine how these rates depend on the degree to which the field is concentrated into $\mathrm{kG}$ bundles (Sect. 4.2) and on the mean unsigned flux density in the area (Sect. 4.3).

\section{Calculations}

\subsection{Numerical methods}

The numerical simulations were realized with the 3D MHD code STAGGER developed by Galsgaard \& Nordlund (1996). The code solves the time-dependent MHD equations by a sixth-order finite difference scheme using fifth-order interpolations for the spatial derivatives, whereas the time evolution is done using a third order Runge-Kutta scheme. For every time step, the radiative transfer equation is solved at every grid point assuming local thermal equilibrium. This is done by using a Feautrier-like scheme along the rays with two $\mu$-angles plus the vertical and four $\phi$-angles horizontally, which add up to nine angles in total. The wavelength dependence of the absorption coefficient was taken into account by the opacity binning method (Nordlund 1982; Skartlien 2000). The equation-of-state table used for all simulations, except the $200 \mathrm{G}, 500 \mathrm{G}$, and $1000 \mathrm{G}$ setup, was calculated using a standard program for ionization equilibria and absorption coefficients (Gustafsson 1973). For the $200 \mathrm{G}, 500 \mathrm{G}$, and $1000 \mathrm{G}$ setups the equation-of-state table used was the one described in Beeck et al. (2012). The horizontal boundaries were periodic, whereas on the top and bottom we had open transmitting boundaries. The effective temperature of the surface was controlled in the standard way (Galsgaard \& Nordlund 1996) by adjusting the entropy of inflowing material at the lower boundary. The magnetic field is kept vertical at the lower boundary. At the top, a potential field extrapolation was implemented as a boundary condition.

\subsection{Setup}

As mentioned in the introduction, observations show that magnetic fields of different polarities do not always tend to diffuse and cancel out, rather only under certain conditions. As argued in Sect. 2 above, the rate of flux canceling seen at the surface must depend on the conditions found deeper down in the convection zone.

We tested how the time scale of the diffusion process changes depending on how deep the magnetic field lines are anchored in the convection zone. The field was allowed to move horizontally at the lower boundary of the simulation box. The anchoring effect was studied by simply varying the depth of this boundary. The effect that we find thus includes only the magnetic connection down to this depth. By omitting magnetic stresses due to the continuation of the field into even deeper layers, we yield a lower bound on the anchoring effect.
We choose two setups, with vertical extents of $10 \mathrm{Mm}$ and of $2.85 \mathrm{Mm}$ measured from the photosphere downwards into the convection zone. In both of these we tested whether the diffusion coefficient changes when the magnetic field is already fragmented into $\mathrm{kG}$ flux tubes before starting the diffusion process. These fragmented magnetic field simulations are compared with those in which the absolute value of the field strength is initially uniform. A third variable whose effect we tested was the strength (average flux density) of the initial field. In all cases, the initial field varies across the box in a pattern of stripes of alternating polarity.

\subsubsection{Uniform initial $\left|B_{z}\right|$ of $20 \mathrm{G}, 50 \mathrm{G}$ and $100 \mathrm{G}$}

For the simulations with an initially uniform vertical magnetic field strength of $B_{z}=50 \mathrm{G}$, the simulations were run in a box of $48 \mathrm{Mm} \times 6 \mathrm{Mm}$ in the horizontal and $3.32 \mathrm{Mm}$ or $10.5 \mathrm{Mm}$, respectively, in the vertical direction. The box in both cases extends to $474 \mathrm{~km}$ above the photosphere. We ran each of these simulations for two different horizontal resolutions, $25 \mathrm{~km}$ and $100 \mathrm{~km}$. The vertical resolution was the same in both cases, varying from a maximum resolution of $11 \mathrm{~km}$ around the photosphere to $34 \mathrm{~km}$ at $3 \mathrm{Mm}$ depth and $55 \mathrm{~km}$ at $10 \mathrm{Mm}$ depth. We used the result of a standard field-free simulation, relaxed to a statistically steady state, as the initial condition for the hydrodynamics. We used two different setups for the initial magnetic field configuration. In one, the magnetic simulation started by adding a vertical magnetic field of uniform strength $\left|B_{z}\right|=50 \mathrm{G}$ in stripes of alternating polarities of $3 \mathrm{Mm}$ width. In the box of $48 \mathrm{Mm}$ this results in sixteen stripes of $3 \mathrm{Mm} \times 6 \mathrm{Mm}$. In the other setup, the stripes of alternating polarity had a width of $6 \mathrm{Mm}$, whereas the vertical magnetic field used was still $\left|B_{z}\right|=50 \mathrm{G}$. The Lorentz force in this field vanishes initially, so that the transition from the hydrodynamic initial condition is smooth. Every simulation ran for $60 \mathrm{~min}$ of solar time. For the simulations with an initial field strength of $\left|B_{z}\right|=20 \mathrm{G}$ and $\left|B_{z}\right|=100 \mathrm{G}$, a similar simulation setup as for the $50 \mathrm{G}$ simulations was used, except that the vertical box size was restricted to a vertical depth of $2.85 \mathrm{Mm}$ and a horizontal resolution of $100 \mathrm{~km}$.

\subsubsection{Initial field fragmented into kilo-gauss fields}

In this setup we first construct a field which is already gathered into magnetic $\mathrm{kG}$ flux tubes before we let the diffusion process set in. This was done with a simulation similar to the above, but for a vertical magnetic field of uniform polarity. The horizontal average vertical field strength was thus constant in time. This was done in a box of $3 \mathrm{Mm} \times 6 \mathrm{Mm}$. This simulation was evolved until the magnetic field had accumulated in the downflows at all depth levels, and a vertical magnetic field strength of at least $1 \mathrm{kG}$ had been reached for some magnetic structures even at the bottom of the simulation box. For the $10.5 \mathrm{Mm}$ simulation this took around 6 hours of solar time and for the $2.85 \mathrm{Mm}$ simulation this took around $2.3 \mathrm{~h}$. The diffusion simulation was then set up by doubling the $3 \times 6 \mathrm{Mm}^{2}$ simulation to a $6 \times 6 \mathrm{Mm}^{2}$ horizontal extent and inverting the magnetic field polarity in one of the $3 \times 6 \mathrm{Mm}^{2}$ stripes. The horizontal resolution was $25 \mathrm{~km}$ and the vertical resolution was as described in Sect. 3.2.1.

In this construction, there are always some field lines that cross the boundary between the two polarities because the horizontal boundary conditions are periodic. The divergence of the magnetic field does not vanish there after the polarity change. The problem can be minimized by moving the boundary at those 

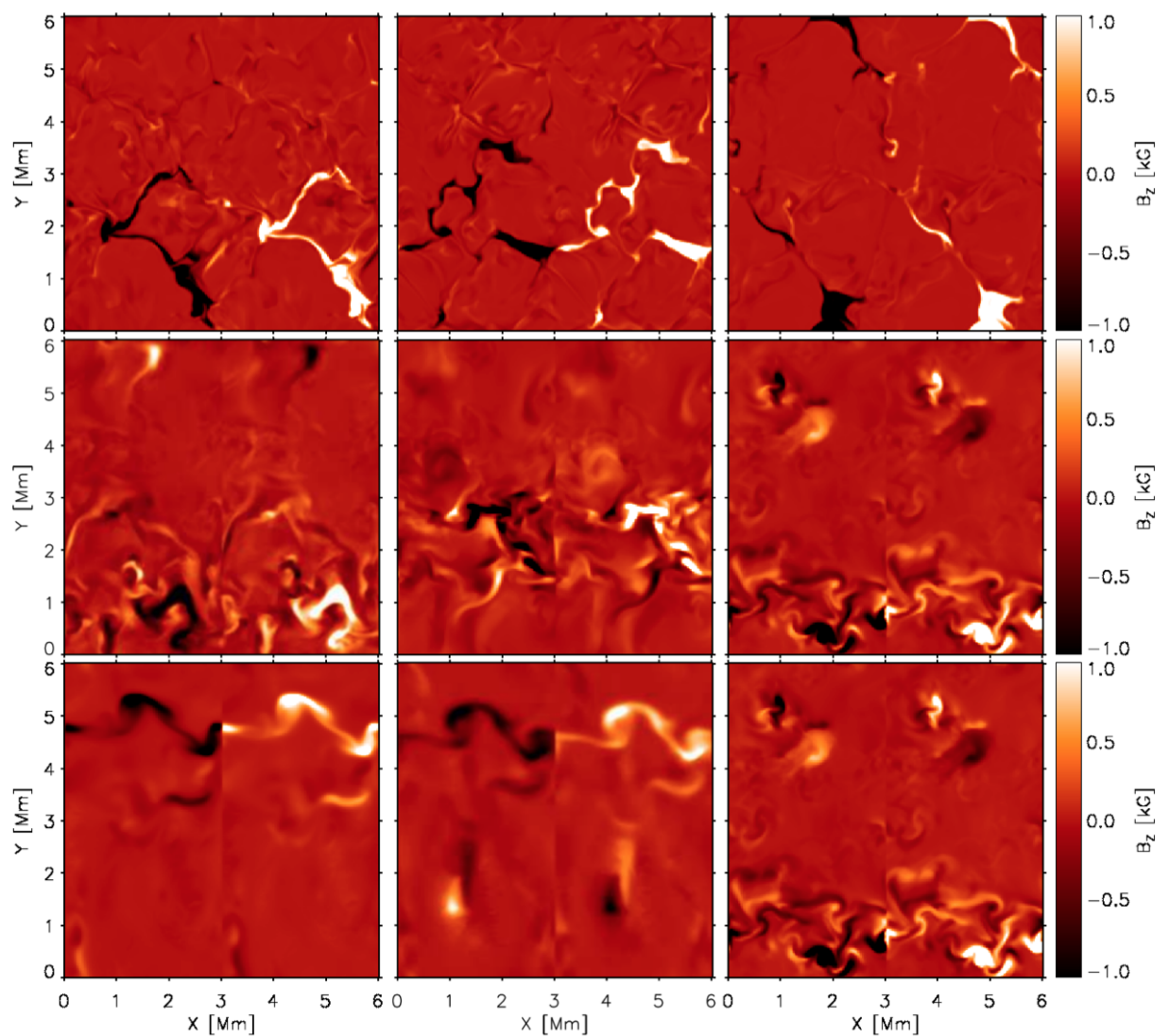

Fig. 1. Vertical magnetic field strength $B_{z}$ of three different initial magnetic field configurations (top from left to right) for simulation runs having an initial magnetic field fragmented into $\mathrm{kG}$ flux tubes before the diffusion process sets in. Horizontal cuts at $z=0$ (top row), $2.85 \mathrm{Mm}$ (middle), and $10 \mathrm{Mm}$ (bottom row). Vertical box depth of the simulation is $10 \mathrm{Mm}$.
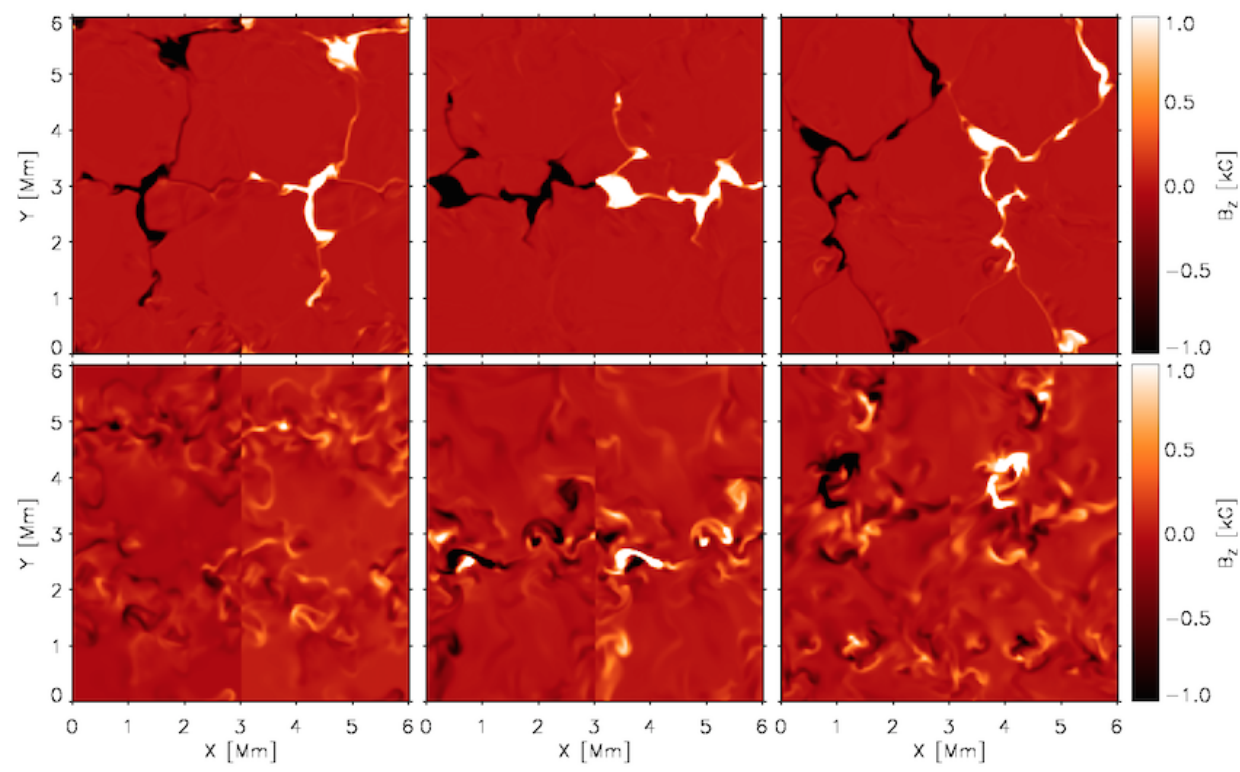

Fig. 2. As per Fig. 1, but for the $2.85 \mathrm{Mm}$ deep simulation. Horizontal cuts at $z=0$ (top row) and $2.85 \mathrm{Mm}$ (bottom).

points where the polarity is changed to a location where it does not cut across a strong magnetic concentration. In addition the $\operatorname{div} \boldsymbol{B}$ cleaning routine of the STAGGER code was used for the first couple of seconds of the simulation.

The initial magnetic field configurations for the $10.5 \mathrm{Mm}-$ deep simulation are shown in Fig. 1 at the nominal depth $z=0$ of the photosphere, and at $2.85 \mathrm{Mm}$ and $10 \mathrm{Mm}$ below the photosphere. The initial states for the $2.85 \mathrm{Mm}$ simulation are shown in Fig. 2 for the photosphere and at $2.85 \mathrm{Mm}$ below the photosphere. In both cases, three different initial conditions are shown, which are used for statistical averaging of the results in the later analysis.

\subsubsection{Uniform initial $\left|B_{z}\right|$ of $200 \mathrm{G}, 500 \mathrm{G}, 1000 \mathrm{G}$}

For measurements of the effective diffusion coefficient dependence on the initial magnetic field strength we used a slightly different setup: we used a box of $12 \times 12 \mathrm{Mm}^{2}$ horizontal and $3.4 \mathrm{Mm}$ depths, $474 \mathrm{~km}$ of which was above the photosphere and $2.7 \mathrm{Mm}$ below. The horizontal resolution was $100 \mathrm{~km}$, whereas the vertical resolution varied from $13 \mathrm{~km}$ near photosphere to $60 \mathrm{~km}$ at the lower boundary. The sign of the initial magnetic field changed in stripes of $3 \mathrm{Mm}$ width, yielding four stripes of $3 \mathrm{Mm} \times 12 \mathrm{Mm}$ of alternating positive and negative polarity. We used setups for three different initial vertical magnetic field 


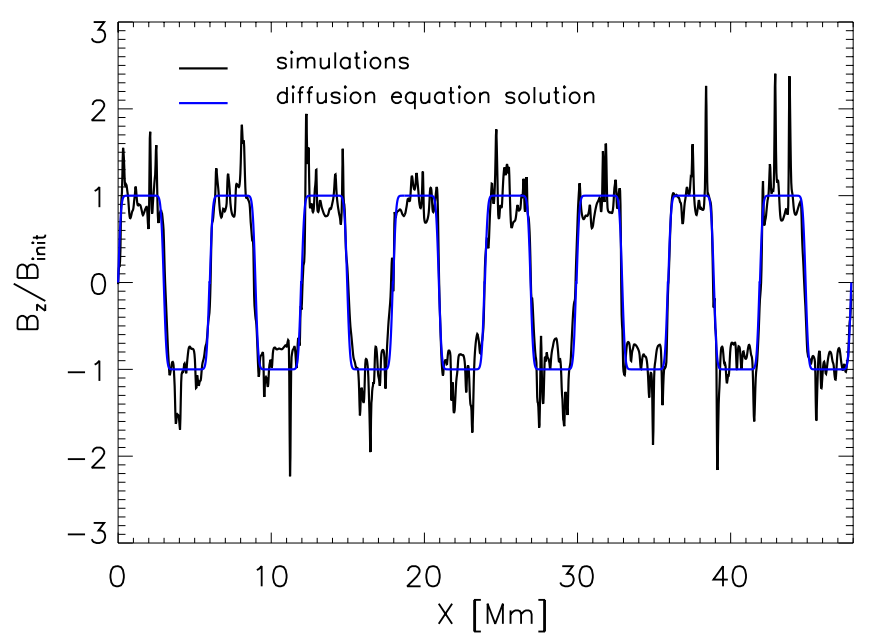

Fig. 3. Square wave after $1.5 \mathrm{~min}$ solar time for a $2.85 \mathrm{Mm}$-deep box and horizontal resolution of $25 \mathrm{~km}$. The best-fit diffusion coefficient is $D=140 \mathrm{~km}^{2} \mathrm{~s}^{-1}$.

strengths: $B_{\text {vert }}=200 \mathrm{G}, 500 \mathrm{G}$, and $1000 \mathrm{G}$. All simulations ran for $60 \mathrm{~min}$ solar time. This setup used an equation-of-state further described in Beeck et al. (2012).

\subsection{Analysis}

The canceling process is studied from the evolution of the vertical component of the magnetic field at the nominal photosphere $z=0$ of the simulations, $B_{z}(x, y, t)$. Where $x$ is the direction in which the polarity of the stripes varies, and $\bar{B}$ is the average in the other horizontal coordinate, $y$ :

$\bar{B}(x, t) \equiv\left\langle B_{z}\right\rangle_{y}$.

By gradual mixing of opposite polarities as well as by actual canceling (Sect. 2.1), the profile of $\bar{B}$ as a function of $x$ smears out with time and decays in amplitude. An effective diffusivity is measured by comparing this with the time dependence of a matching one-dimensional diffusion problem with an assumed constant diffusion coefficient $D$. If $B_{\mathrm{f}}(x, t)$ is the field strength of this matching problem, it is governed by the standard diffusion equation

$\frac{\partial B_{\mathrm{f}}}{\partial t}=D \frac{\partial^{2} B_{\mathrm{f}}}{\partial x^{2}}$

The initial condition is the striped pattern used in the simulations, with amplitude $B_{0}$ and period of length $L$, which contains two adjacent stripes of opposite polarity and has an origin $x-0$ at the boundary between polarities. The solution of Eq. (2) is then

$B_{\mathrm{f}}=\frac{4}{\pi} B_{0} \sum_{n=1}^{\infty} \frac{1}{2 n-1} \sin \left(k_{n} x\right) \mathrm{e}^{-k_{n}^{2} D t}$,

where

$k_{n}=(2 n-1) 2 \pi / L$.

This was compared with $\bar{B}$ determined from the simulations and to determine the best-fitting value of $D$. This value is a function of $t$ because of the statistical fluctuations in the simulations, but more importantly because the evolution of the field is not a simple 2D diffusion process. In addition to a different time dependence, the measured values of $D$ are expected to depend on the

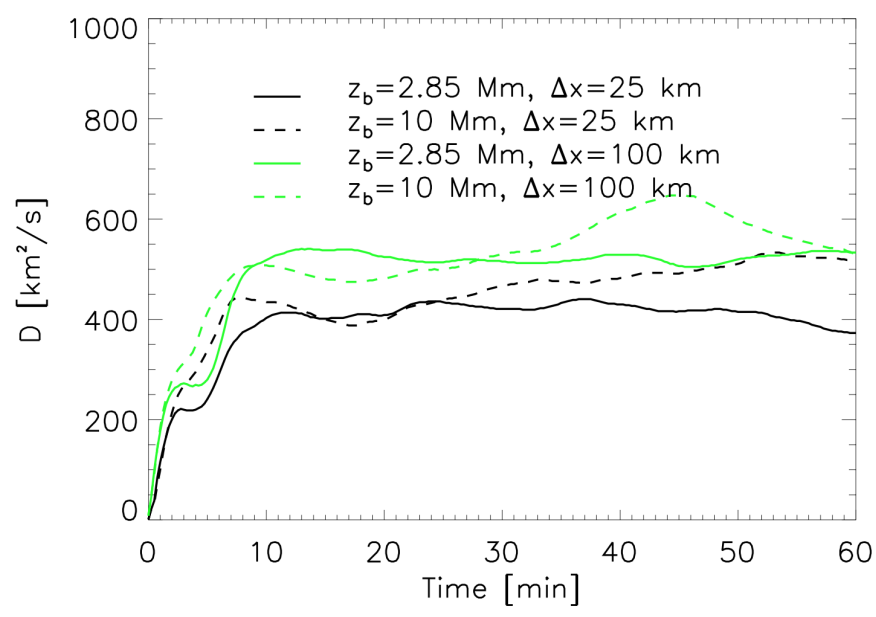

Fig. 4. Effective diffusion coefficient measured by fit to Eq. (3), (direct analysis method). Vertical initial field $\left|B_{z}\right|=$ cst. $=50 \mathrm{G}$. Dashed: box depth $10 \mathrm{Mm}$, solid: box depth $2.85 \mathrm{Mm}$. Green: horizontal resolution $100 \mathrm{~km}$, black: horizontal resolution $25 \mathrm{~km}$.

depth $z_{\mathrm{b}}$ of the lower boundary of the simulations and amplitude $B_{0}$ of the initial flux density.

The process of determining best-fit values of $D\left(t, z_{\mathrm{b}}, B_{0}\right)$ was done in two different ways. In the straightforward way, which we call "direct", the values of $D(t)$ are determined by matching the profile of $B_{\mathrm{f}}(x)$ to that of $\bar{B}(x)$ by a least-squares process. An example of the resulting best fit is shown in Fig. 3 .

In the "indirect" method, absolute values $|B|$ are used for the comparison. Their integral over $x$ declines with time due to mixing and canceling of polarities. The comparison with the analytic model yields values for $D(t)$. This process is not exactly equivalent to the direct method. The action of convective flows produces an initial amplification of the field through the wrapping up of field lines. This adds some small-scale flux of mixed opposite polarity. The amplification process does not change the net flux through the surface, hence it cancels out in the direct method. However, it has to be accounted for in the indirect method.

\section{Results}

\subsection{Initial vertical magnetic field with uniform $\left|B_{z}\right|=50 \mathrm{G}$}

\subsubsection{Direct analysis}

We applied the direct analysis method to the simulation runs with lower boundary at $2.85 \mathrm{Mm}$ and $10 \mathrm{Mm}$ depth, as described in Sect. 3.2.1. We started from uniform vertical magnetic field of $50 \mathrm{G}$, alternating the polarity in stripes of $3 \mathrm{Mm}$ width. Figure 4 shows the resulting effective diffusion coefficients for the horizontal resolutions of $25 \mathrm{~km}$ (black lines) and $100 \mathrm{~km}$ (green lines) and the two different box depths. After an initial transient of about $10 \mathrm{~min}$, which can be attributed to the magnetic field amplification process due to turbulent stretching, the measured effective diffusion coefficient stays almost constant for all simulation runs. The values range from $400-600 \mathrm{~km}^{2} \mathrm{~s}^{-1}$. The effective coefficients we obtain for the simulation run with the higher horizontal resolution of $25 \mathrm{~km}$ are slightly lower than those for the horizontal resolution of $100 \mathrm{~km}$. This shows that at $100 \mathrm{~km}$ resolution numerical diffusion still plays a role. The depth of the simulation box does not seem to have a strong influence on the effective coefficients measured at the photosphere. It is not surprising that the diffusion coefficients at deeper layers look very 


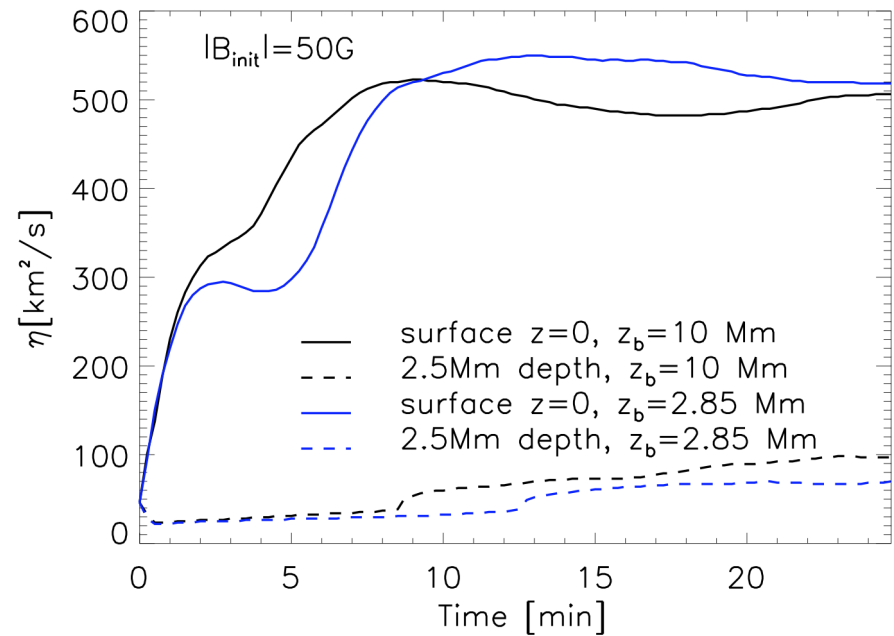

Fig. 5. Diffusion coefficients calculated for two depth levels using the direct analysis method. Solid: the photosphere, dashed: at $2.5 \mathrm{Mm}$ depth. This is shown for two simulation runs with different vertical box sizes: blue: box depth $2.85 \mathrm{Mm}$, black: box depth $10 \mathrm{Mm}$.

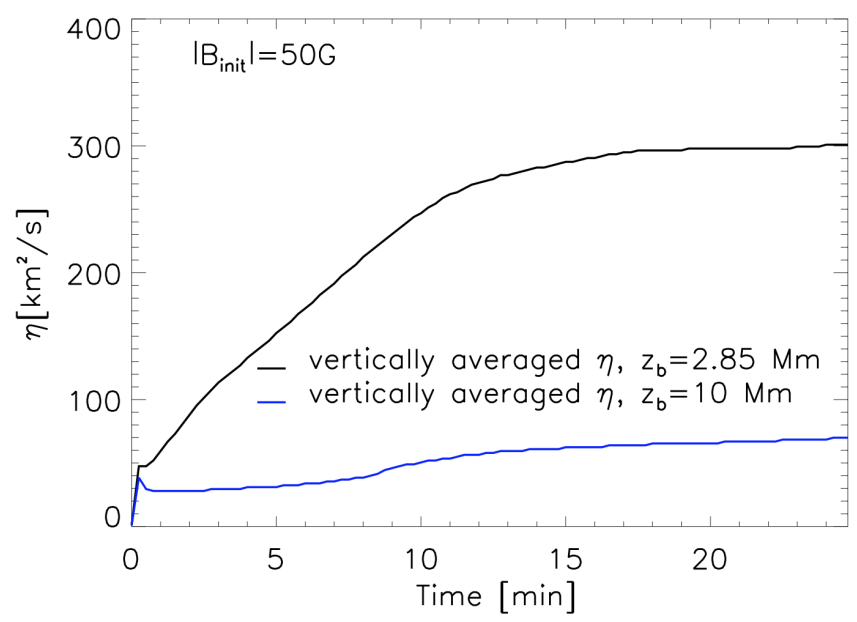

Fig. 6. Vertically-averaged diffusion coefficients calculated using the direct analysis method. Black: box depth $2.85 \mathrm{Mm}$, blue: box depth $10 \mathrm{Mm}$.

much alike as well, independent of the vertical extent of the simulation box. This is indicated by Fig. 5, which shows the diffusion coefficients at the surface and at $2.5 \mathrm{Mm}$ depth. However, vertically averaged magnetic field diffusion coefficients for different vertical box extensions differ (Fig. 6).

\subsubsection{Indirect analysis}

The evolution of the absolute value of the vertical component, $\left|B_{z}\right|$ is determined not only by the diffusion process, but also by the magnetic field strength amplification due to turbulent flows. To quantify this effect, and to compensate for it, a comparison simulation was run for an initially uniform vertical field without polarity changes, but otherwise identical conditions. The evolution of the surface average of $\left\langle\left|B_{z}\right|\right\rangle_{\mathrm{u}}$ in this simulation is shown in Fig. 7. As expected from interaction with the convective flow, the amplification effect initially increases rapidly and then saturates after a few granule turnover times.

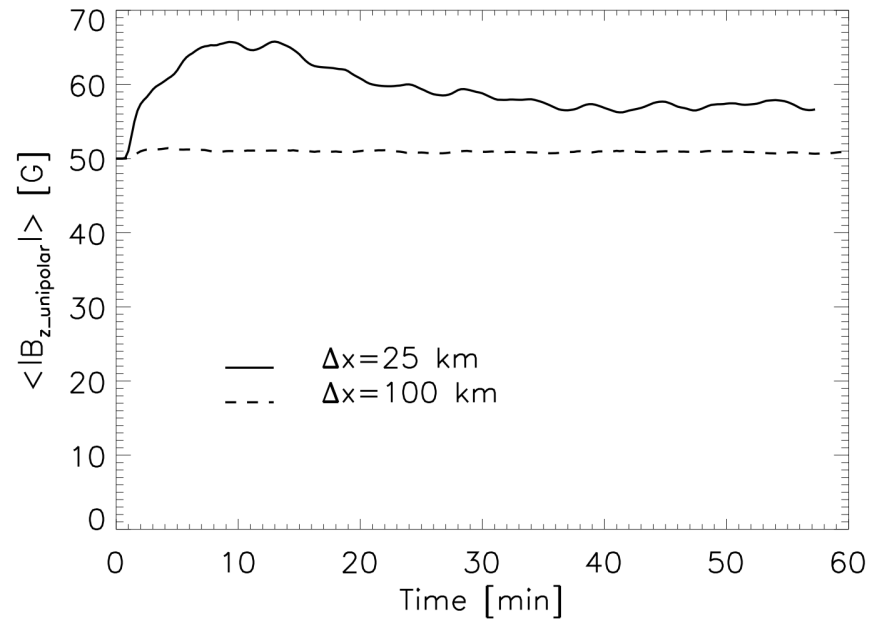

Fig. 7. Amplification of the mean unsigned flux density by field line stretching, in an initially uniform vertical field of $50 \mathrm{G}$.

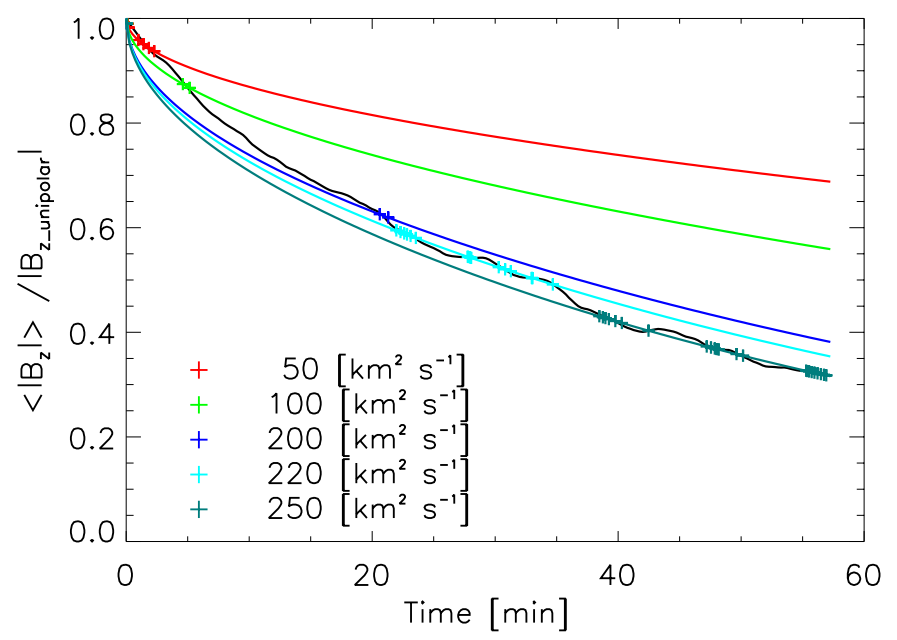

Fig. 8. Measurement of effective diffusion coefficients by the indirect method. Black: time evolution of the average vertical field strength for a box depth of $2.85 \mathrm{Mm}$. Initial magnetic field $\left|B_{z}\right|=50 \mathrm{G}$, polarity stripe width $3 \mathrm{Mm}$, horizontal resolution $25 \mathrm{~km}$. Colors: Eq. (3) for five values of the assumed diffusion coefficient. Symbols mark the intersection points with the numerical evolution shown in Fig. 11.

As long as the field is sufficiently weak, the amplification is a multiplicative effect, meaning it is proportional to the initial value. Assuming this to be the case, the corresponding diffusion simulation can be corrected simply for this effect by dividing the mean $\left\langle\left|B_{z}\right|\right\rangle$ by the value $\left\langle\left|B_{z}\right|\right\rangle_{\mathrm{u}}$ from the comparison.

Figure 8 shows the evolution of $\left\langle\left|B_{z}\right|\right\rangle$ for the simulation with the lower boundary $z_{\mathrm{b}}=2.85 \mathrm{Mm}$. Overplotted are the analytic solutions for five different values of the diffusion coefficient. The diffusion coefficients that match vary between $50-250 \mathrm{~km}^{2} \mathrm{~s}^{-1}$. Simulations with $z_{\mathrm{b}}=10 \mathrm{Mm}$ depth yield similar values, 20$250 \mathrm{~km}^{2} \mathrm{~s}^{-1}$. In comparison to the diffusion coefficients obtained with the direct analysis, these are about a factor of two lower. At the lower horizontal resolution of $100 \mathrm{~km}$, the measurements yield values of $250-400 \mathrm{~km}^{2} \mathrm{~s}^{-1}$ for both depths of the simulation box (Fig. 9), which is a factor of 1.3 lower than using the direct analysis method. We attribute this to the small-scale mixed-polarity fields produced during the evolution of the imposed initial field (Fig. 7). Because the indirect method is based on the absolute value of $B_{z}$, it contains a component that is absent 


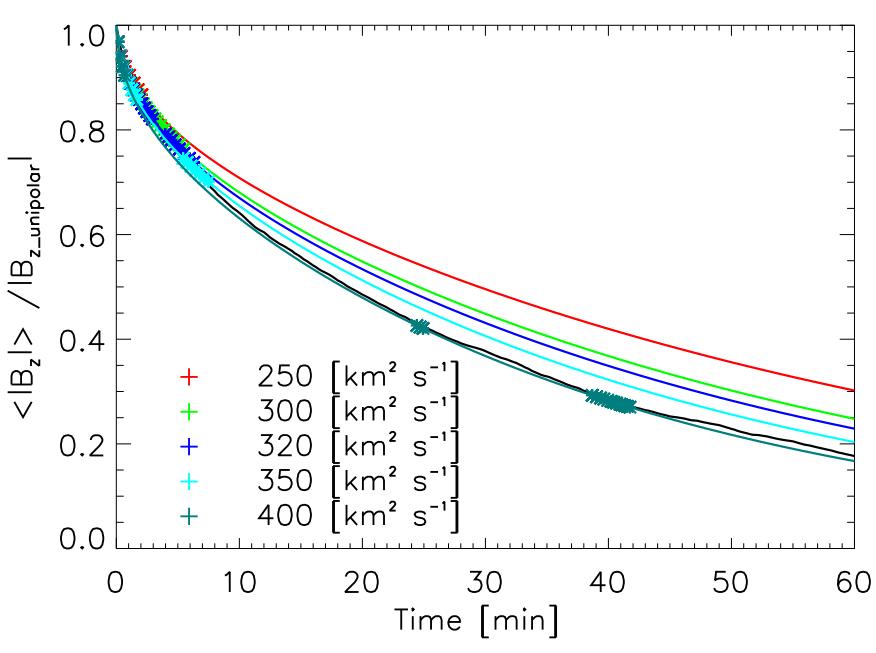

Fig. 9. As per Fig. 8, but for a horizontal resolution of $100 \mathrm{~km}$.

in the direct measurement. In the indirect method this component is absent initally but adds to the measured flux at later times and decreases the apparent decay rate.

The fact that the depth of the simulation box does not seem to play a role for the diffusion coefficients obtained, at least for the unfragmented simulations, did not match with our expectations. This is because the freedom of the magnetic field lines to move around should be increasingly limited the deeper down they are anchored in the convection zone. This lead us to conclude that the spatial scales at which the restoring force would be felt strongly enough are larger than the $3 \mathrm{Mm}$ width of the stripes with which we simulated the diffusion process. Therefore we additionally analyzed, as shown in Fig. 10, the time evolution of the horizontally-averaged vertical magnetic field $\left\langle\left|B_{z}\right|\right\rangle$ at the photosphere for a unipolar stripe length of $6 \mathrm{Mm}(L=12 \mathrm{Mm})$ for vertical extents below the photosphere of $2.85 \mathrm{Mm}$ and $10 \mathrm{Mm}$. For comparison, the case of a unipolar stripe length of $3 \mathrm{Mm}$ $(L=6 \mathrm{Mm})$ is also shown. However, no significant difference is seen between the $L=12 \mathrm{Mm}$ simulation runs for a vertical box extent of $10 \mathrm{Mm}$ and $2.85 \mathrm{Mm}$, leading in both cases to effective diffusion coefficients of $D=300-600 \mathrm{~km}^{2} \mathrm{~s}^{-1}$. The decay timescales seen in Fig. 10 are about three times longer for $L=12 \mathrm{Mm}$ than for $L=6 \mathrm{Mm}$, instead of the factor of four predicted by a simple diffusion model.

A simple estimate of the timescales involved may give a good explanation for these results. For a magnetic element sitting in the center of a unipolar stripe of $3 \mathrm{Mm}$ size, the distance to be traveled to meet magnetic concentrations of the opposite polarity is of the order of $1.5 \mathrm{Mm}$ when neglecting the fact that the other polarity is also approaching, and the horizontal convective velocities at the photosphere are of the order of $1 \mathrm{~km} \mathrm{~s}^{-1}$. Therefore we assume that the travel time to meet the different polarity flux is approximately $20 \mathrm{~min}$. We furthermore assume that the field strength in the flux tube is in the order of $\mathrm{kG}$, which leads to a mean Alfvén speed of around $1 \mathrm{~km} \mathrm{~s}^{-1}$. If magnetic field lines advected by convective flows are anchored at a depth of $10 \mathrm{Mm}$, the time it takes for this information to travel from the photosphere to $10 \mathrm{Mm}$ depth and back would then be approximately five hours. This rough estimate shows that for a diffusion process acting in relatively small unipolar regions, the anchoring of the field lines should not play a major role.

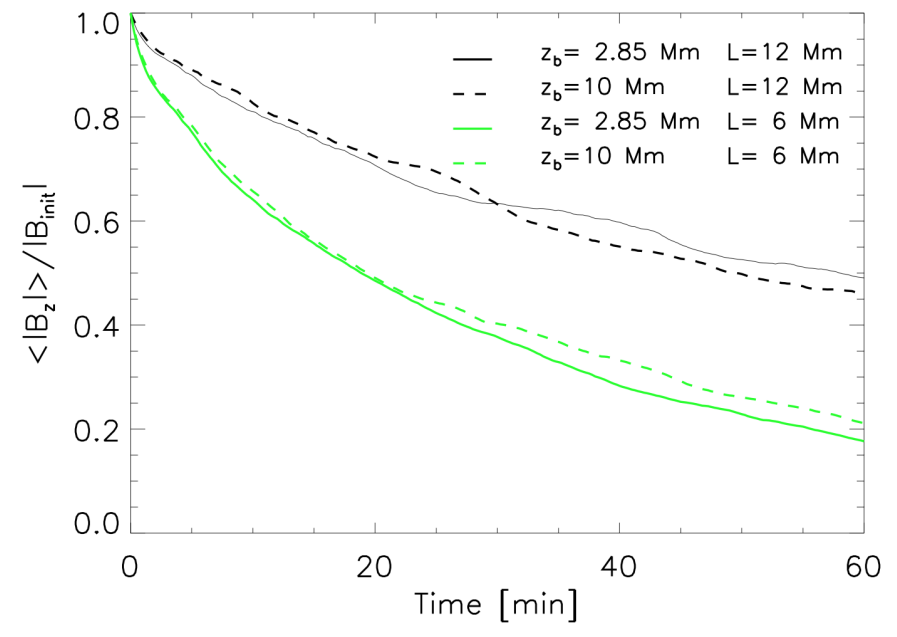

Fig. 10. Comparison of $\left\langle\left|B_{z}\right|\right\rangle$ for a unipolar stripe length of $3 \mathrm{Mm}$ (green, $L=6 \mathrm{Mm}$ ) versus $6 \mathrm{Mm}$ (black, $L=12 \mathrm{Mm}$ ). Solid: box depth $2.85 \mathrm{Mm}$, dashed: box depth $10 \mathrm{Mm}$.

\subsection{Initial magnetic field fragmented into $k G$ flux tubes}

Figure 11 compares results for initial conditions in which the magnetic field is already fragmented into $\mathrm{kG}$ flux tubes and those from the uniform initial condition. For the time evolution of the fragmented simulations, an average of the three magnetic field representations pictured in Figs. 1 and 2 has been taken. Apart from the different initial conditions, the simulations are the same as before. The fragmented simulations are normalized by their initial field strength, whereas the non-fragmented simulations are normalized by their unipolar equivalent to correct for the turbulent amplification component (as described above). Figure 11 shows that the depth of the simulation box does not appear to have a major influence upon the range of parameters used in these simulations.

The diffusion coefficients measured with the indirect method in the fragmented magnetic field simulation range from 20$120 \mathrm{~km}^{2} \mathrm{~s}^{-1}$ for a box depth of $2.85 \mathrm{Mm}$, whereas for a $10 \mathrm{Mm}$ box depth they range from $20-60 \mathrm{~km}^{2} \mathrm{~s}^{-1}$ (Fig. 12). The diffusion coefficients for a $10 \mathrm{Mm}$ box depth are a bit lower than the ones for a $3 \mathrm{Mm}$ box depth. It is not clear if this can be interpreted as an indicator for a depth dependence, because the statistical fluctuations due to the small box sizes are still large. Nevertheless the values of the diffusion coefficients derived from the fragmented magnetic field simulations are about $100 \mathrm{~km}^{2} \mathrm{~s}^{-1}$ lower than for the uniform magnetic field configuration case, which brings them in the range of values observationally obtained for granular flows $\left(60-90 \mathrm{~km}^{2} \mathrm{~s}^{-1}\right)$.

\subsection{Dependence on initial field strength}

To test the dependence of the effective diffusion coefficients on field strength, we used simulations with initially vertical fields $\left|B_{\text {init }}\right|$ of uniform strength ranging from $20-1000 \mathrm{G}$. The pattern of alternating polarity stripes of $3 \mathrm{Mm}$ is used as before (Sects. 3.2.1 and 3.2.3). Figure 13 shows the decay of the average surface field strength $\left|B_{z}\right|(t)$. Figure 14 displays the results for $\left|B_{\text {init }}\right|=500 \mathrm{G}$ using the indirect analysis method, which leads to values in the range $D=500-830 \mathrm{~km}^{2} \mathrm{~s}^{-1}$ within the first $60 \mathrm{~min}$ of solar time. Comparing this to Fig. 8 shows that the diffusion coefficients for $\left|B_{\text {init }}\right|=500 \mathrm{G}$ are about a factor of two higher than in the $50 \mathrm{G}$ case $\left(50-250 \mathrm{~km}^{2} \mathrm{~s}^{-1}\right)$. The striking dependence 


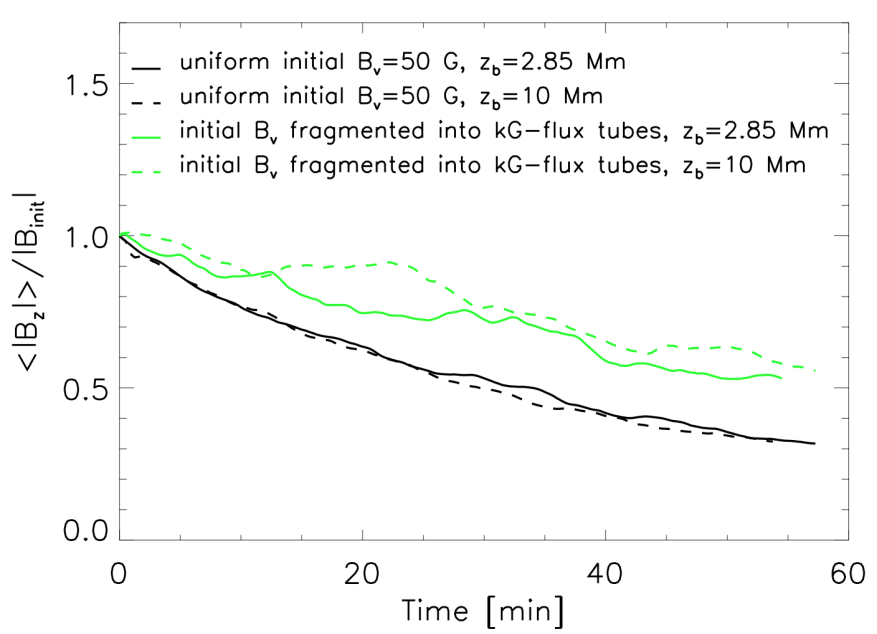

Fig. 11. Evolution of the mean flux density at the surface for different initial magnetic field configurations and vertical box sizes. Dashed: box depth $10 \mathrm{Mm}$, solid: box depth $2.85 \mathrm{Mm}$. Green: initial magnetic field fragmented into $\mathrm{kG}$ flux tubes. Black: uniform magnetic field configuration of $50 \mathrm{G}$. Curves corrected for convective amplification effect (as detailed in the text).

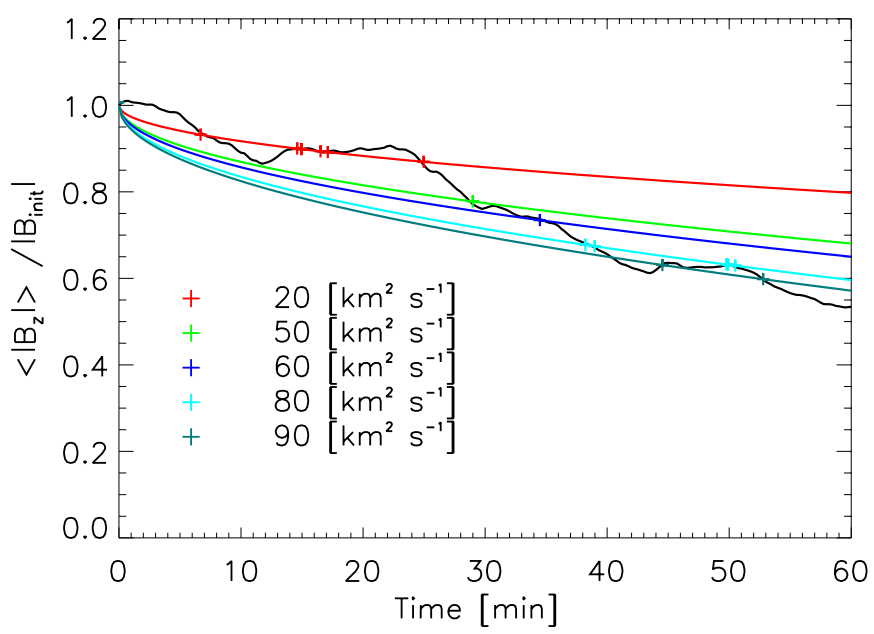

Fig. 12. As per Fig. 8, but for a box depth of $10 \mathrm{Mm}$.

on field strength can also be seen directly in Fig. 13. The decay times of $\left|B_{z}\right|$ to $50 \%$ of $\left|B_{\text {init }}\right|$ are $[25,19,15,10,7$, and 3$] \mathrm{min}$ for $\left|B_{\text {init }}\right|=[20,50,100,200,500$, and 1000$] \mathrm{G}$, respectively.

The direct and indirect analysis yield similar results (comparing Fig. 14 to Fig. 15). Figure 15 also illustrates that in the case of $\left|B_{\text {init }}\right|=1000 \mathrm{G}$ the peak effective diffusion coefficient reaches $2600 \mathrm{~km}^{2} \mathrm{~s}^{-1}$ during the first couple of minutes. The other simulations clearly show that higher initial field strengths lead to higher initial effective diffusion coefficients.

A simple diffusion model would predict that the diffusion process is independent of the initial field strength, which is clearly not the case here. Comparison of $\left|B_{\text {init }}\right|=20 \mathrm{G}$ with the $50 \mathrm{G}$ case shows that a noticeable dependence is present already at a field strength of approximately $50 \mathrm{G}$, which marks the conventional definition of the boundary of active regions. As discussed in Sect. 2.1.2, this can be attributed to the dominance of the magnetic field energy density in the atmosphere. The analogy would be to two parallel bar magnets of opposite polarities, where one pole of each is covered by the plasma of the convection zone, whereas the other pole reaches up through

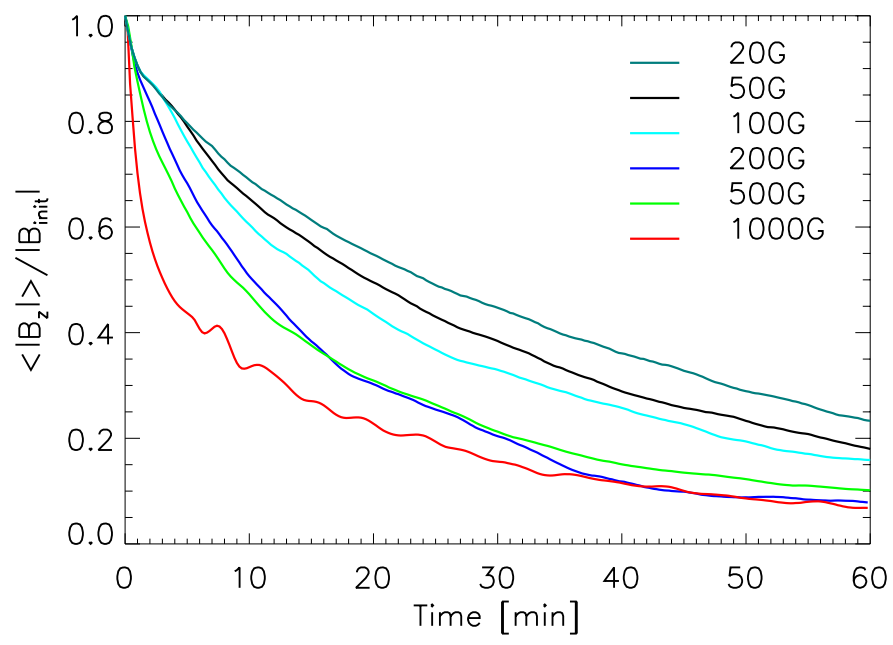

Fig. 13. Evolution of average surface field strength $\left\langle\left|B_{z}\right|\right\rangle(t)$ for $B_{\text {init }}$ of $20 \mathrm{G}$ (blue-green), $50 \mathrm{G}$ (black), $100 \mathrm{G}$ (cyan), $200 \mathrm{G}$ (blue), $500 \mathrm{G}$ (green) and $1000 \mathrm{G}$ (red). Polarity stripe width $3 \mathrm{Mm}$, a box depth of $2.85 \mathrm{Mm} / 2.7 \mathrm{Mm}$.

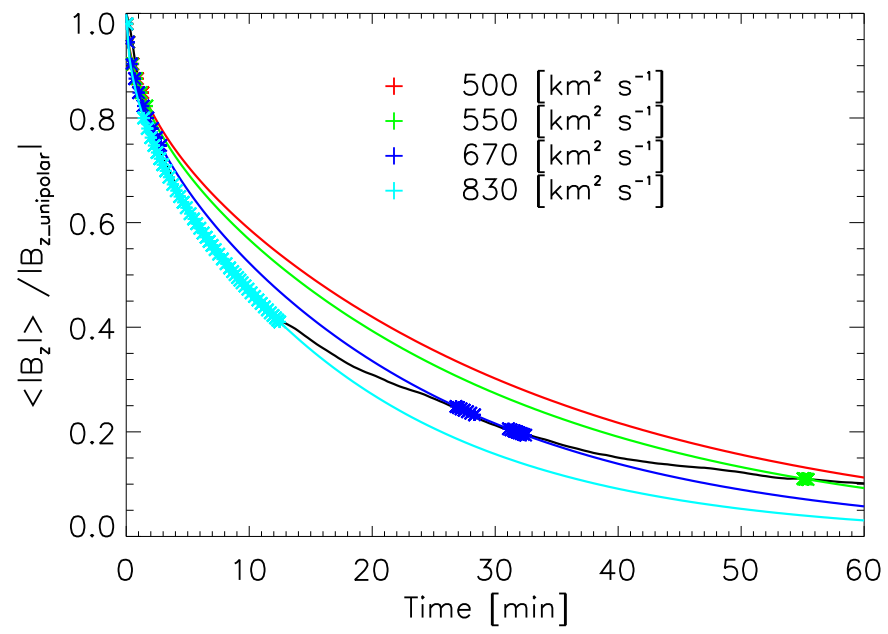

Fig. 14. Determination of effective diffusion coefficients with the indirect analysis method for an initially uniform vertical magnetic field of $500 \mathrm{G}$ in a $2.7 \mathrm{Mm}$ vertically-extended simulation.

the photosphere. Above the surface the opposite polarities attract each other, but the parts buried in the convection zone do not feel each other due to the plasma shielding. Apparently (Fig. 13) a field strength of $200 \mathrm{G}$ is high enough for this effect to play a dominant role in the retraction process.

\subsection{The retraction process}

The retraction process is illustrated in Fig. 16, showing the time evolution of one selected field line for the $200 \mathrm{G}$ simulation (top), the $500 \mathrm{G}$ simulation (middle) and the $1000 \mathrm{G}$ simulation (bottom). To obtain this $2 \mathrm{D}$ view from the $3 \mathrm{D}$ field configuration, the magnetic field was averaged along the horizontal direction that is perpendicular to the plane of the figure. In this $2 \mathrm{D}$ projection a stream function, the value of which identifies individual field lines, can be calculated for the field vector. To connect this function between consecutive time steps, the displacement of a fluid element on the field line between time steps is deduced from the vertical velocity of the fluid. The value of the stream function at 


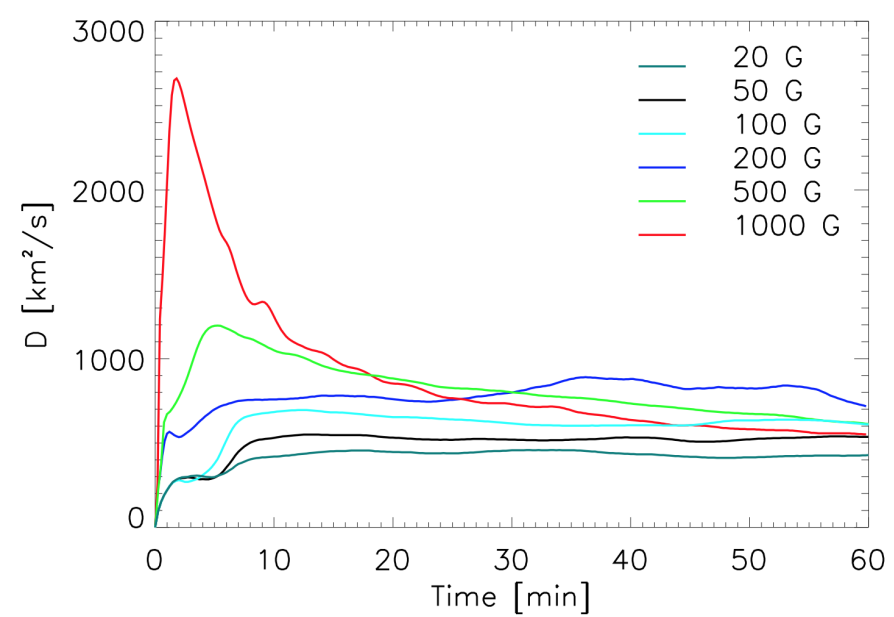

Fig. 15. Time dependence of effective diffusion coefficients determined with the direct analysis method, from the data in Fig. 13.

the new time and position then identifies the entire field line at the new time.

It is clear that retraction plays a role for all three depicted initial field strengths during the first few minutes of the diffusion process. Its magnitude is evidently defined by the magnetic field strength, such that the highest downflow velocities are observed for the $1000 \mathrm{G}$ simulation run and the lowest for the $200 \mathrm{G}$ simulation. This is in agreement with Fig. 15.

A better illustration of the retraction signatures is given in Figs. 17-20. These show the difference between the bipolar and the unipolar simulations for several variables and time steps for an initial field strength of $50 \mathrm{G}, 200 \mathrm{G}, 500 \mathrm{G}$ and $1000 \mathrm{G}$. The top panels show the vertical velocity differences, the middle panels the horizontal velocity differences, and the bottom panels the temperature differences. Within each panel the plots correspond, from top to bottom, to snapshots at times $t=60 \mathrm{~s}, 135 \mathrm{~s}$ and $210 \mathrm{~s}$. Figures $17-20$ show clear downflow velocity signatures at the boundaries between the unipolar stripes, that increase in magnitude with increasing initial field strength. The strong downflows are accompanied by horizontal inflows, as expected for a retraction process. A clear initial heating above the photosphere is associated with the retraction process (lower rows in Figs. 17-20). This is due to the stability of the radiative stratification as above $z=0$ the entropy of the atmosphere increases with height. Compared with the mean atmosphere, the retracting downflow heats up by compression. As the Figs. 17-20 show, this heating stops once the field has retracted below the surface and the surface flow gets replaced by normal granulation. These model results suggest that for $\mathrm{kG}$ field strengths the retraction process could produce a local temperature increase of the same order of magnitude $\left(\Delta T \approx 10^{3} \mathrm{~K}\right)$ as discussed for Ellerman bombs (Georgoulis 2002; Fang et al. 2006; Kitai 1983), even though the underlying physical process is different. The downflow velocities associated with Ellerman bombs on the photospheric level seem to be a by a factor ten lower $\left(\sim 0.1-0.3 \mathrm{~km} \mathrm{~s}^{-1}\right.$, Georgoulis 2002), than we see here in our simulations.

\section{Discussion and conclusions}

The most surprising result we find, when studying magnetic flux canceling with 3D MHD simulations, is the strong dependence of the decay rate of the average surface field on the initial vertical field strength. An initially uniform magnetic field of $500 \mathrm{G}$
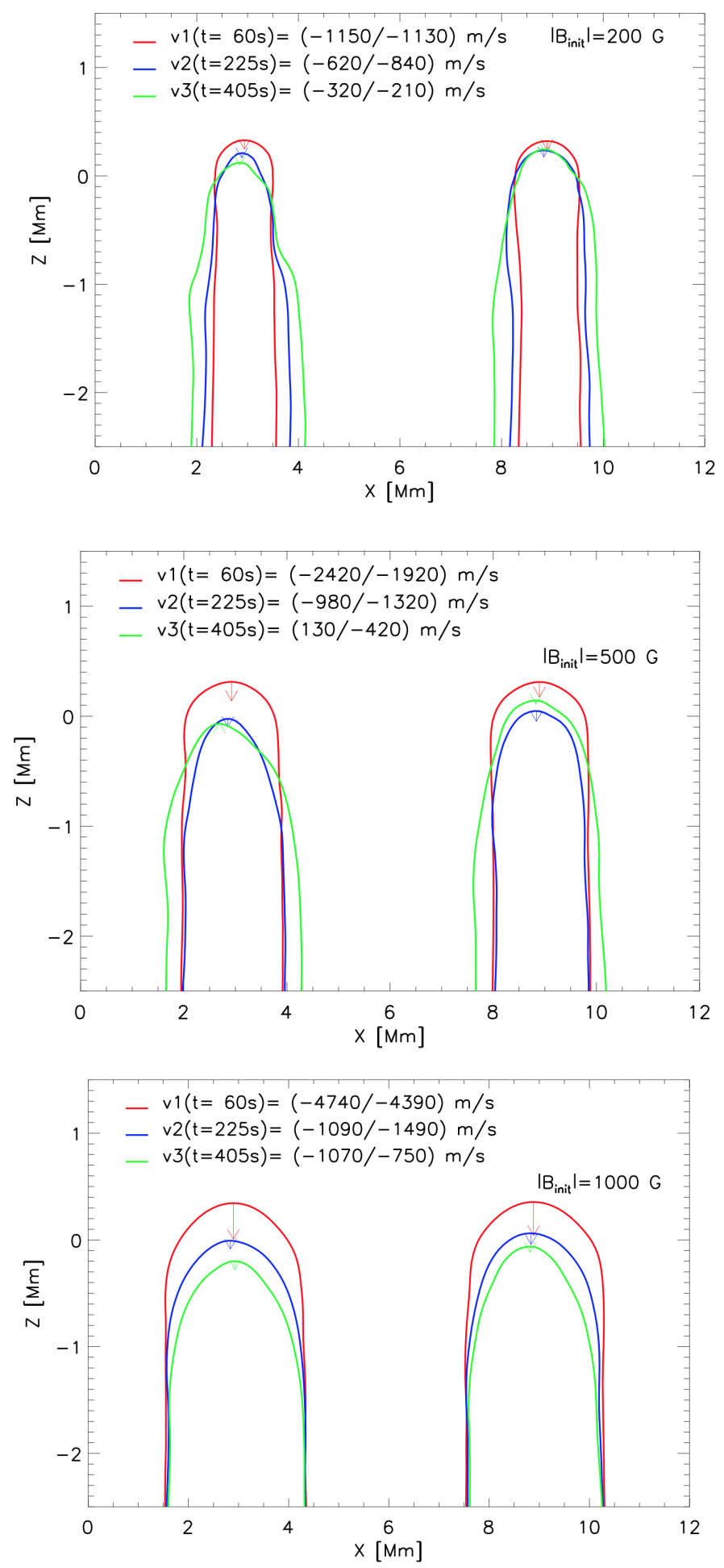

Fig. 16. Field lines from the $200 \mathrm{G}$ simulation (top), $500 \mathrm{G}$ (middle) and $1000 \mathrm{G}$ simulation (bottom), after $t=60 \mathrm{~s}$ (red), $225 \mathrm{~s}$ (blue) and $405 \mathrm{~s}$ (green).

in stripes of alternating polarity shows an initial decay rate that is about a factor of two higher than that obtained using an initial field strength of $50 \mathrm{G}$. This deviation decreases asymptotically over time and approaches the diffusion coefficient from the $50 \mathrm{G}$ setup after about $60 \mathrm{~min}$ of simulation time. This magnetic field strength dependence is even more pronounced for an initial magnetic field strength of $1 \mathrm{kG}$, for which the diffusion coefficient is 

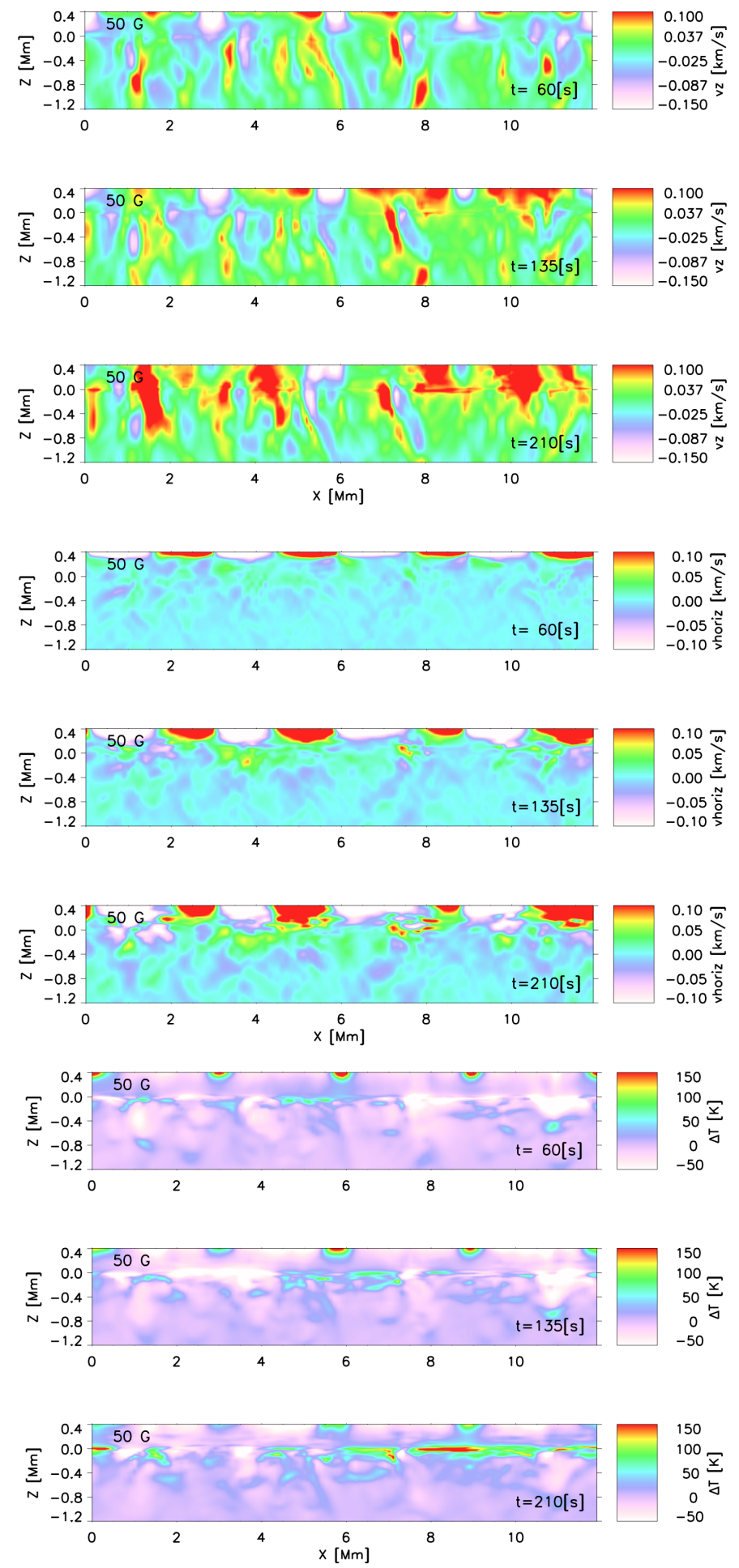

Fig. 17. Difference between the simulation run with alternating magnetic polarity stripes and the unipolar magnetic field simulation for an initial field strength of $50 \mathrm{G}$. Top, middle, bottom plot in each panel correspond to $t=60 \mathrm{~s}, 135 \mathrm{~s}$ and $t=210 \mathrm{~s}$ respectively. Top panel: vertical velocity difference in $\mathrm{km} \mathrm{s}^{-1}$, middle panel: horizontal velocity difference in $\mathrm{km} \mathrm{s}^{-1}$, bottom panel: temperature difference.

initially a factor of five higher than for the $50 \mathrm{G}$ simulation. This behavior is explained by the mutual attraction of the different field polarities that enhance the canceling rate according to their field strength. We find clear signatures of this retraction process
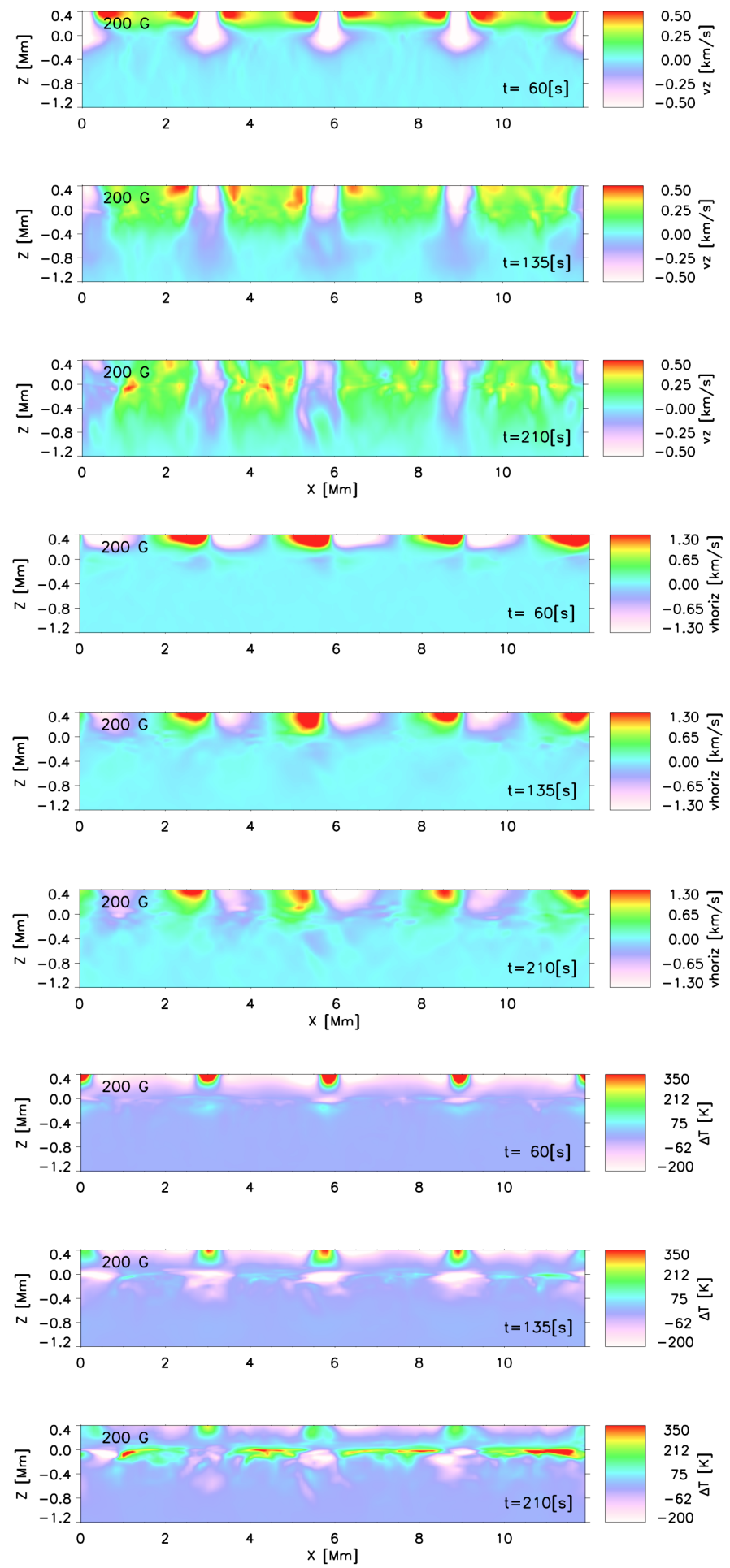

Fig. 18. As per Fig. 17 but for $200 \mathrm{G}$ initial field strength.

in our simulations, and find that its strength depends on the initial field strength. Strong downflows accompanied by horizontal inflows were detected at the boundaries of the opposite polarity regions. These were associated with an initial heating process, wherein the retraction downflow heated up the mean atmosphere by compression. The heating stopped once the field had retracted below the surface. The detected process plausibly explains the rapid retraction of magnetic flux observed in active-region complexes. 

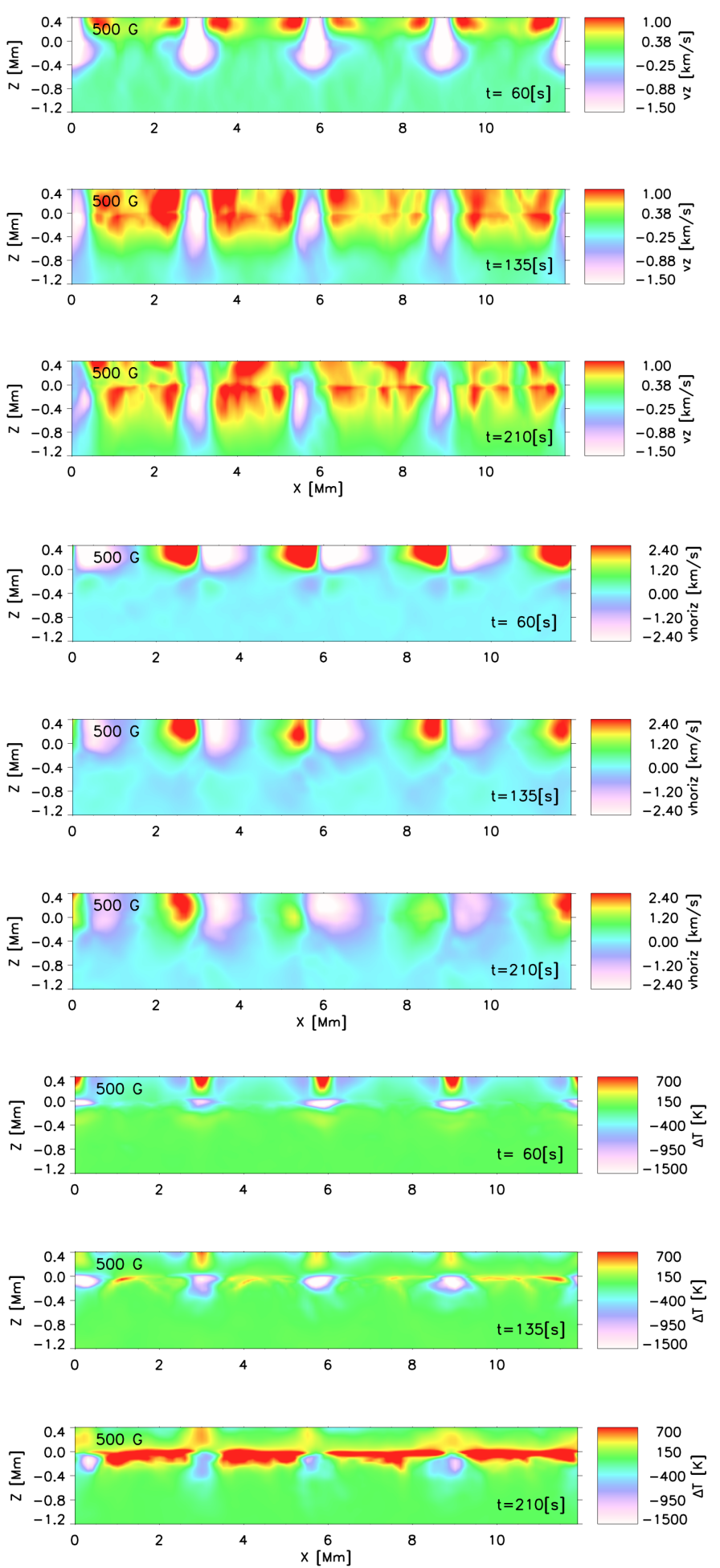

Fig. 19. As per Fig. 17 but for $500 \mathrm{G}$ initial field strength.

One of the other questions addressed with these simulations is how the effective "cancellation" seen at the surface in a mixture of polarities depends on the magnetic field configuration deeper down in the convection zone. To test this, the vertical extent of the simulation box was varied, expecting that the deeper down in the convection zone the magnetic field lines were anchored, the more rigid they would act and the more the diffusion process would be delayed. However, the effective diffusion coefficients from the simulations for different vertical depth but the
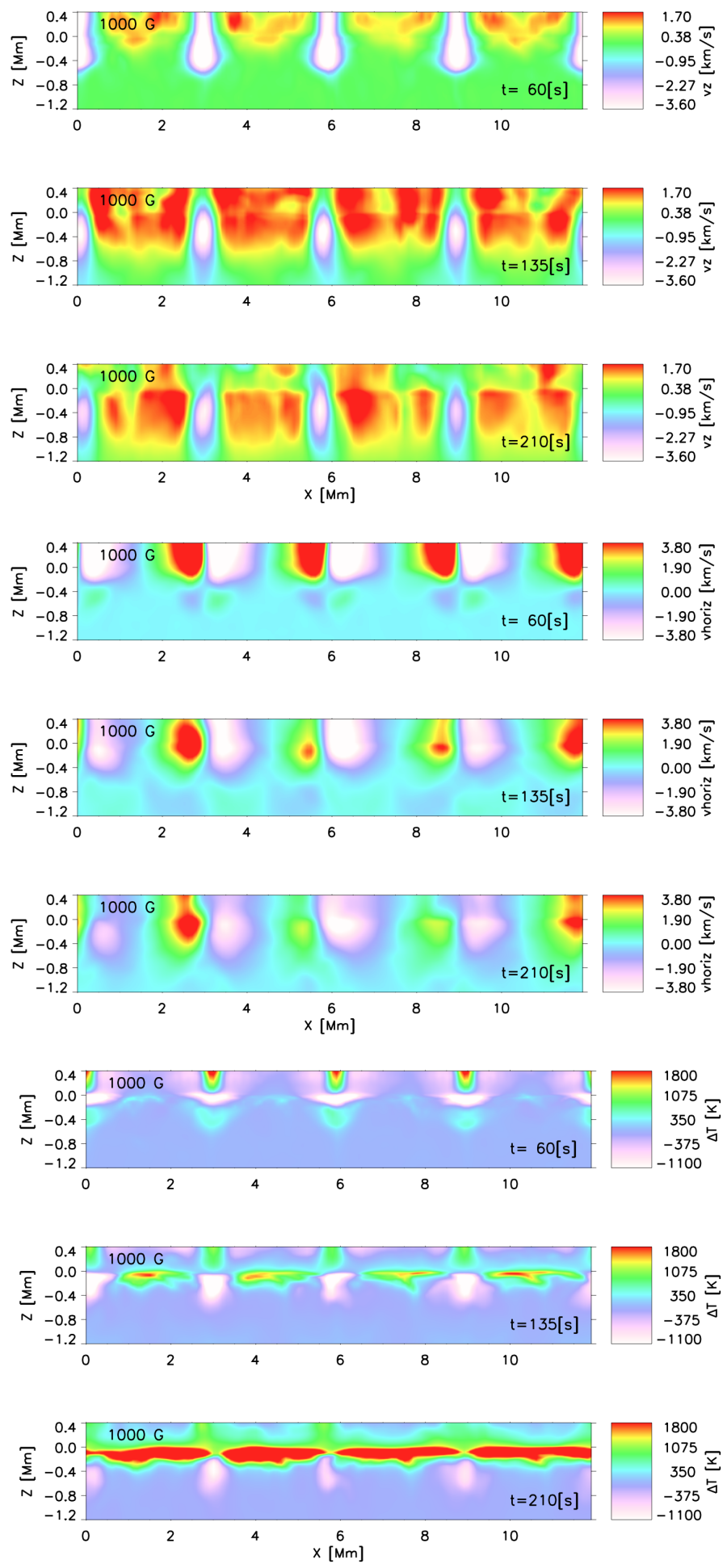

Fig. 20. As per Fig. 17 but for $1000 \mathrm{G}$ initial field strength.

same unipolar stripe length are essentially the same. This was tested for box extents of $2.85 \mathrm{Mm}$ and $10 \mathrm{Mm}$ depth below the photosphere and unipolar stripe lengths of $3 \mathrm{Mm} \times 6 \mathrm{Mm}$ as well as for $6 \mathrm{Mm} \times 6 \mathrm{Mm}$ ones.

It seems that the timescales at which the restoring force acts are too long, so that the different polarities have already canceled and retracted below the surface before the magnetic tension forces have had time to propagate up from the deeper layers. Nevertheless, what we find is a dependence on the fragmentation 
level of the magnetic field. In other words if the magnetic field is fragmented into $\mathrm{kG}$ flux tubes before the diffusion process starts, the diffusion process works slower. As a result, the diffusion values shift from those obtained from the uniform initial field configuration $300-600 \mathrm{~km}^{2} \mathrm{~s}^{-1}$, to those better matching the values obtained observationally for granular flows, $20-120 \mathrm{~km}^{2} \mathrm{~s}^{-1}$. For simulations with a $10 \mathrm{Mm}$ depth and a initially fragmented field, slightly lower diffusion coefficients were derived compared to the simulations with a $2.85 \mathrm{Mm}$ depth, but this could be due to statistical noise.

As a word of caution, the evolution of fields of mixed polarity as measured here by "effective diffusion coefficients" is not to be interpreted as justification for replacing the evolution of actual MHD flows by "turbulent diffusion". The magnetic field follows the MHD induction equation, and does not behave like a passive scalar. It cannot be represented by the evolution of a scalar (cf. Hotta et al. 2012), even if the field were weak enough to be advected passively. Because of the Lorentz force it is actually not passive either, at least not in the present context. It is not a surprise, then, that fitting the evolution of the field by a passive scalar on a $2 \mathrm{D}$ surface produces widely varying values of the diffusion coefficient, including negative values. These differences, rather than the values themselves, are in fact the best source of information on the evolution of the magnetic field, in particular in the layers below the surface. For example, if the surface field is anchored to deeper layers, diffusion over larger distances is limited by the strength of the connecting field, and its evolution is instead dictated at the anchoring depth rather than by near-surface flows.

Acknowledgements. Thanks to Åke Nordlund and the anonymous referee for the very helpful comments and suggestions which led to a major improvement of the paper. The research leading to these results received funding from the European Research Council under the European Unionâ Seventh Framework Program (FP/2007-2013)/ERC Grant Agreement No. 307117.

\section{References}

Abramenko, V. I., Carbone, V., Yurchyshyn, V., et al. 2011, ApJ, 743, 133

Afram, N., Unruh, Y. C., Solanki, S. K., et al. 2011, A\&A, 526, A120

Beeck, B., Collet, R., Steffen, M., et al. 2012, A\&A, 539, A121

Berger, T. E., Löfdahl, M. G., Shine, R. A., \& Title, A. M. 1998, ApJ, 506, 439

Bray, R. J., \& Loughhead, R. E. 1984, Science, 226, 571

Cameron, R., Vögler, A., \& Schüssler, M. 2011, A\&A, 533, A86

Chae, J., Litvinenko, Y. E., \& Sakurai, T. 2008, ApJ, 683, 1153

Cheung, M. C. M., Schüssler, M., Tarbell, T. D., \& Title, A. M. 2008, ApJ, 687, 1373

Chitta, L. P., Kariyappa, R., van Ballegooijen, A. A., DeLuca, E. E., \& Solanki, S. K. 2014, ApJ, 793, 112

Galsgaard, K., \& Nordlund, Å. 1996, J. Geophys. Res., 101, 13445

Georgoulis, M. K., Rust, D. M., Bernasconi, P. N., \& Schmieder, B. 2002, ApJ 575,506
Gustafsson, B. 1973, Uppsala Astron. Obs. Ann., 5, 6

Fang, C., Tang, Y. H., Xu, Z., Ding, M. D., \& Chen, P. F. 2006, ApJ., 643, 1325 Hagenaar, H. J., Schrijver, C. J., Title, A. M., \& Shine, R. A. 1999, ApJ, 511, 932

Hotta, H., Iida, Y., \& Yokoyama, T. 2012, ApJ, 751, L9

Howard, R., \& Labonte, B. J. 1981, Sol. Phys., 74, 131

Isobe, H., \& Shibata, K. 2004, in The Solar-B Mission and the Forefront of Solar Physics, ASP Conf. Ser., 325, 63

Jiang, J., Hathaway, D. H., Cameron, R. H., et al. 2014, Space Sci. Rev., 186, 491

Kálmán, B. 2001, A\&A, 371, 731

Kubo, M., Low, B. C., \& Lites, B. W. 2010a, ApJ, 712, 1321

Kubo, M., Chye Low, B., \& Lites, B. 2010b, 38th COSPAR Scientific Assembly, 38,2828

Kubo, M., Low, B. C., \& Lites, B. W. 2014, ApJ, 793, 9

Lamb, D. A., DeForest, C. E., Hagenaar, H. J., Parnell, C. E., \& Welsch, B. T. 2010, ApJ, 720, 1405

Komm, R. W., Howard, R. F., \& Harvey, J. W. 1995, Sol. Phys., 158, 213

Kitai, R. 1983, Sol. Phys., 87, 135

Kubat, J., \& Karlicky, M. 1986, Bull. Astron. Institutes of Czechoslovakia, 37, 155

Leighton, R. B. 1969, ApJ, 156, 1

Martin, S. F. 1990, in Solar Photosphere: Structure, Convection, and Magnetic Fields, IAU Symp., 138, 129

Martin, S. F., Livi, S. H. B., \& Wang, J. 1985, Austr. J. Phys., 38, 929

Moreno-Insertis, F., Caligari, P., \& Schüssler, M. 1994, Sol. Phys., 153, 449

Mosher, J. M. 1977, Ph.D. Thesis, California Institute of Technology, Pasadena

Nordlund, A. 1982, A\&A, 107, 1

Peter, H., Tian, H., Curdt, W., et al. 2014, Science, 346, 6207

Petrovay, K., \& Moreno-Insertis, F. 1997, ApJ, 485, 398

Rabin, D., Moore, R., \& Hagyard, M. J. 1984, ApJ, 287, 404

Rempel, M., \& Cheung, M. C. M. 2014, ApJ, 785, 90

Rüdiger, G., \& Kitchatinov, L. L. 2000, Astron. Nachr., 321, 75

Sheeley, N. R. 1992, in The solar cycle, ed. K. L. Harvey, San Francisco, ASP Conf. Ser., 27, 562

Smithson, R. C. 1973, Sol. Phys., 29, 365

Schrijver, C. J., \& Martin, S. F. 1990, Sol. Phys., 129, 95

Shibata, K., Tajima, T., Steinolfson, R. S., \& Matsumoto, R. 1989, ApJ, 345, 584

Skartlien, R. 2000, ApJ, 536, 465

Spruit, H. C. 2013, Essential magnetohydrodynamics for astrohysics [arXiv: 1301.5572v2]

Spruit, H. C., \& Zweibel, E. G. 1979, Sol. Phys., 62, 15

Stein, R. F., \& Nordlund, Å. 2006, ApJ, 642, 1246

Strous, L. H., Scharmer, G., Tarbell, T. D., Title, A. M., \& Zwaan, C. 1996, A\&A, 306, 947

Tobias, S. M., \& Cattaneo, F. 2013, J. Fluid Mech., 717, 347

Topka, K. P., Tarbell, T. D., \& Title, A. M. 1986, ApJ, 306, 304

Vissers, G. J. M., Rouppe van der Voort, L. H. M., \& Rutten, R. J. 2013, ApJ, 774,32

Vrabec, D. 1974, in Chromospheric Fine Structure, ed. R. G. Athay, IAU Symp., 56,201

Wang, H. 1988, Sol. Phys., 117, 343

Wang, Y.-M., Sheeley, N. R., Jr., \& Nash, A. G. 1991, ApJ, 383, 431

Watanabe, H., Vissers, G., Kitai, R., Rouppe van der Voort, L., \& Rutten, R. J. 2011, ApJ, 71

Yang, S., Zhang, J., Li, T., \& Liu, Y. 2012, ApJ, 752, L24

Zwaan, C. 1978, Sol. Phys., 60, 213

Zwaan, C. 1985, Sol. Phys., 100, 397

Zwaan, C. 1987, ARA\&A, 25, 83 